\title{
PI3K inhibitor LY294002 inhibits activation of the Akt/mTOR pathway induced by an oncolytic adenovirus expressing TRAIL and sensitizes multiple myeloma cells to the oncolytic virus
}

\author{
YIN TONG $^{1 *}$, WEIWEI ZHU ${ }^{1 *}$, XIANBO HUANG ${ }^{1}$, LIANGSHUN YOU $^{1}$, \\ XIUJUN HAN ${ }^{2}$, CHUNMEI YANG ${ }^{1}$ and WENBIN QIAN ${ }^{1}$ \\ ${ }^{1}$ Institute of Hematology, The First Affiliated Hospital, College of Medicine, Zhejiang University, Hangzhou 310003; \\ ${ }^{2}$ Women's Hospital, College of Medicine, Zhejiang University, Hangzhou 310006, P.R. China
}

Received October 23, 2013; Accepted November 18, 2013

DOI: 10.3892/or.2014.3020

\begin{abstract}
Recently, much progress has been achieved in the treatment of multiple myeloma (MM). However, the major challenge of chemotherapeutic drugs is acquired resistance. Oncolytic virotherapies offer promising alternatives; with the possibility of their integration with current therapeutic strategies. In the present study, we assessed the potential of ZD55-TRAIL (an oncolytic adenovirus expressing tumor necrosis factor-related apoptosis-inducing ligand) as an oncolytic agent for MM. Our results clearly indicated that ZD55 armed with TRAIL was more cytotoxic to drug-sensitive as well as drug-resistant MM cell lines, than the virus alone. Furthermore, it was also observed that ZD55-TRAIL induced apoptosis through the activation of the caspase pathway. In particular, ZD55-TRAIL significantly inhibited insulin-like grow th factor-1 receptor (IGF-1R) and NFאB. However, IGF did not abrogate ZD55-TRAIL-induced cell death. Combination of ZD55-TRAIL with the PI3K inhibitor LY294002 in RPMI-8226 cells inhibited the virus-mediated activation of mTOR and AKT, thus, promoting cell death. Combined treatment of ZD55-TRAIL and MG132 (a proteasome inhibitor) robustly increased the expression of death receptor 5 (DR5), which enhanced the apoptosis response without significant toxicity to normal liver cells. Collectively, our results suggest that combined treatment of TRAIL-armed oncolytic adenovirus and a PI3K inhibitor or a proteosome inhibitor may serve as a promising therapy for MM.
\end{abstract}

Correspondence to: Professor Wenbin Qian, Institute of Hematology, The First Affiliated Hospital, College of Medicine, Zhejiang University, Qingchun Road 79, Hangzhou 310003, P.R. China

E-mail: qianwenb@hotmail.com

${ }^{*}$ Contributed equally

Key words: multiple myeloma, oncolysis, TRAIL, insulin-like growth factor-1 receptor, mTOR, AKT, apoptosis

\section{Introduction}

Multiple myeloma (MM) is an incurable lymphoid cancer of terminally differentiated B-cell lineage or plasma cells that accounts for $1 \%$ of all cancers and slightly more than $10 \%$ of all hematological malignancies (1). Current treatment for MM, including proteasome inhibitors, immunomodulatory drugs, high-dose chemotherapy and stem cell transplantation, have significantly prolonged the 10 -year overall survival $(2,3)$. However, patients presenting with relapse are numerous due to drug-resistant disease and minimal residual disease (4), necessitating the development of new therapeutic approaches.

Tumor necrosis factor (TNF)-related apoptosis-inducing ligand (TRAIL, Apo-2L) is one of the members of the TNF gene superfamily that selectively induces apoptosis of cancer cells but not of normal cells in various tissue types and is currently in clinical trials for the treatment of human cancers $(5,6)$. Since $\mathrm{MM}$ is characterized as a tumor composed of long-surviving rather than fast-growing malignant plasma cells, potentiating apoptosis has been envisaged as a potential therapeutic approach (7). Previous studies found that exogenous TRAIL induced apoptosis in MM cells from patients, one of which had chemotherapy-refractory disease and in many MM cell lines, including cells sensitive or resistant to dexamethasone (Dex), doxorubicin (Dox), melphalan and mitoxantrone $(8,9)$. This indicates that TRAIL appears effective at inducing apoptosis in chemo-resistant MM cells. Moreover, it has been shown that chemotherapeutic drugs such as Dox can sensitize MM cells to TRAIL through upregulation of death receptor 4 (DR4) and death receptor 5 (DR5) (10). Notably, a recent study revealed that TRAIL, used in combination with Dox, eliminated tumor-initiating cells resulting in complete and lasting eradication of MM tumors in vivo (11). Taken together, these observations indicate that TRAIL-based therapy represents a novel and effective approach for the treatment of human MM.

Cancer targeting gene-virotherapy is a novel therapeutic platform for cancer (12-14). This strategy involves the use of oncolytic adenoviruses (OAs) engineered to express a therapeutic gene inserted into the E1 or E3 region of the viruses, in which the input amount of transgene is amplified by selective replication of the viruses, resulting in longer transgene 
expression within tumor cells and potent activity against tumors. For example, it was reported that TRAIL expression following gene transfer of OAs in human cancer cell lines was 4-fold higher than that obtained with a replication-defective adenovirus (14). We previously constructed the oncolytic adenoviral vector ZD55, an E1B-55K deleted OA, carrying TRAIL (ZD55-TRAIL) (15) and demonstrated that the combination of TRAIL and viral oncolysis yielded an additive cytotoxic effect on cancer cells in vitro. This virus was more effective than the unarmed control at killing tumors in vivo and prolonging survival in murine models $(15,16)$. These findings prompted us to study the effect of ZD55-TRAIL on MM cell lines. In the present study, we found that ZD55-TRAIL was effective against MM cell lines including a drug-resistant cell line and inhibited levels of insulin-like growth factor-1 receptor (IGF-1R). However, the activation of AKT and mTOR was observed upon virus infection. Based on this finding, we investigated the synergistic effects of ZD55-TRAIL combined with the PI3K inhibitor LY294002. This combination therapy significantly improved the sensitivity of MM cells to ZD55-TRAIL. Furthermore, treatment of MM cells with ZD55-TRAIL in combination with MG132, a proteasome inhibitor, resulted in enhanced apoptosis, which was related, at least in part, to upregulation of DR5. Together, the findings of the present study suggest that combination therapy with ZD55TRAIL and a PI3K inhibitor or MG132 may be particularly appealing in the context of drug-resistant MM.

\section{Materials and methods}

Cell culture and reagents. Cell culture medium RPMI-1640 and fetal bovine serum (FBS) were purchased from HyClone Laboratories (Logan, UT, USA). Human MM cell line RPMI-8226 was purchased from the American Type Culture Collection (Rockville, MD, USA). Dex-sensitive (MM.1S) and Dex-resistant (MM1.R) cell lines were kindly provided by Dr Steven Rosen (Northwestern University, Chicago, IL, USA). L-02, a normal human liver cell line, was obtained from the Shanghai Cell Collection (Shanghai, China). The reagents used in the present study were purchased from the following vendors: interleukin-6 (IL-6) and IGF-1 (both from Peprotech, Inc., Rocky Hill, NJ, USA); LY294002 and MG132 (Sigma, St. Louis, MO, USA). Oncolytic viruses including ZD55-TRAIL, ZD55-EGFP and ZD55 were generous gifts from Professor Xinyuan Liu (Xinyuan Institute of Medicine and Biotechnology, School of Life Sciences, Zhejiang University of Technology and Sciences, Hangzhou, China).

Cell viability (MTT) assay. Cell viability was determined by the MTT-based cytotoxicity assay (Sigma), as previously described (16). Briefly, cells were treated with various concentrations of the oncolytic virus ZD55 or ZD55-TRAIL for $48 \mathrm{~h}$. On termination, the medium was replaced with fresh medium containing $0.5 \mathrm{mg} / \mathrm{ml}$ MTT. The cells were incubated for an additional $4 \mathrm{~h}$ at $37^{\circ} \mathrm{C}$. Following removal of the medium and MTT, DMSO $(200 \mu \mathrm{l})$ was added to each well, and the absorbance at $570 \mathrm{~nm}$ was detected.

Colony forming cell assay. MM colony formation assay was performed as described elsewhere (17). Briefly, RPMI-8226 cells $\left(2 \times 10^{3}\right.$ cells $)$ were cultured with IMDM medium containing FBS $(200 \mu \mathrm{l})$ and $2.7 \%$ methylcellulose $(300 \mu \mathrm{l}$; Sigma) at $37^{\circ} \mathrm{C}$ in a humidified atmosphere with $5 \% \mathrm{CO}_{2}$. After 7 days, colonies that contained $>50$ cells were scored manually under a light microscope (Olympus, Tokyo, Japan).

Hoechst DNA staining. Nuclei were stained with Hoechst 33258 (Sigma) to detect chromatin condensation and nuclear fragmentation, which are phenotypic characteristics of apoptosis. Treated RPMI-8226 cells were fixed with $4 \%$ paraformaldehyde and then stained with Hoechst $33258(1 \mu \mathrm{g} / \mathrm{ml})$ for $15 \mathrm{~min}$. Stained slides were analyzed under a fluorescence microscope (Olympus).

Western blot analysis. Western blotting was carried out as described previously (17). Briefly, equal amounts of total cell lysates were separated on sodium dodecyl-sulfate (SDS)polyacrylamide gels containing $8-12 \%$ acrylamide gradients and then transferred to polyvinylidene difluoride membranes (Millipore, Bedford, MA, USA). Non-specific binding was blocked with $10 \mathrm{mM}$ Tris-HCL buffered saline plus $0.05 \%$ Tween-20 containing 5\% skimmed milk powder for $1 \mathrm{~h}$ at room temperature. Membranes were incubated with the primary antibody overnight at $4^{\circ} \mathrm{C}$. The primary antibodies used here were as follows: poly[adenosine diphosphate (ADP)-ribose] polymerase (PARP), caspase-8, -9 and -3, Bcl-xL, cytochrome $c$ (cyto $c$ ), p110 $\alpha$, IGF-1R, AKT, p-AKT (Ser473), mTOR, p-mTOR (Ser2448), p-mTOR (Ser2481), p70S6K, p-p70S6K (Thr389), 4E-BP1, p-4E-BP1 (Thr70) and DR5 purchased from Cell Signaling Technology, Inc. (Beverly, CA, USA); DR4 was provided by Bioworld (Louis Park, MN, USA); $\beta$-actin was obtained from Santa Cruz Biotechnology, Inc. (Santa Cruz, CA, USA). TRAIL and p110 were purchased from BioLegend (San Diego, CA, USA) and Epitomics (Burlingame, CA, USA), respectively.

Preparation of $c D N A$ and real-time PCR. Preparation of total RNA was conducted using an RNeasy Plus kit (Takara Shuzo, Kyoto, Japan). Each cDNA template was made from total RNA with the Superscript II reverse transcriptase kit according to the manufacturer's instructions (Invitrogen Life Technologies, Carlsbad, CA, USA). For IGF-1R we used 5'-TTAAAATGGC CAGAACCTGAG-3' as the forward primer, and 5'-ATTATA ACCAAGCCTCCCAC-3' as the reverse primer. For GAPDH we used 5'-GTAACCCGTTGAACCCCATT-3' as the forward primer, and 5'-CCATCCAATCGGTAGTAGCG-3' as the reverse primer. Amplification reactions were performed using SYBR $^{\circledR}$ Premix Ex Taq ${ }^{\text {TM }}$ (Takara Shuzo) in the iQ5 Multicolor Real-time PCR detection system (Bio-Rad, Hercules, CA, USA). Thermal cycling conditions were as follows: $30 \mathrm{sec}$ at $95^{\circ} \mathrm{C}$ for initial denaturing, $5 \mathrm{sec}$ at $95^{\circ} \mathrm{C}$ for denaturing and $30 \mathrm{sec}$ at $60^{\circ} \mathrm{C}$ for annealing and extension for a total of 40 cycles. The fold-change in mRNA was calculated by the $2^{-\Delta \Delta \mathrm{Ct}}$ method.

Statistical analysis. Statistical analysis of the experimental results was conducted by the Student's t-test. A P-value $<0.05$ was considered to indicate a statistically significant result. Synergisms in the combination treatments were analyzed using CalcuSyn software (Biosoft, Cambridge, UK). 
A
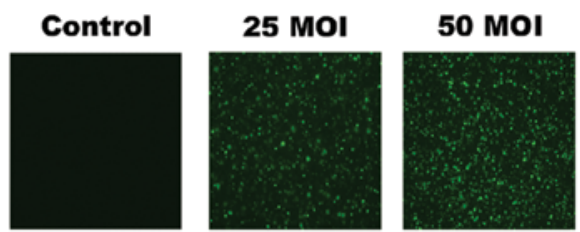

B

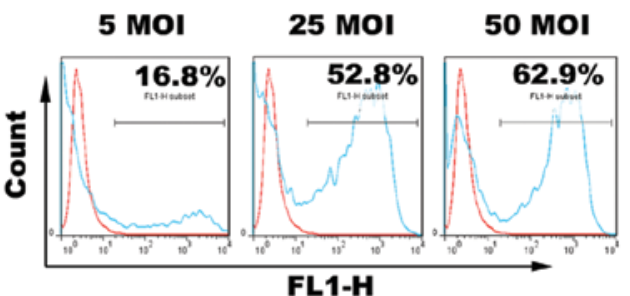

C

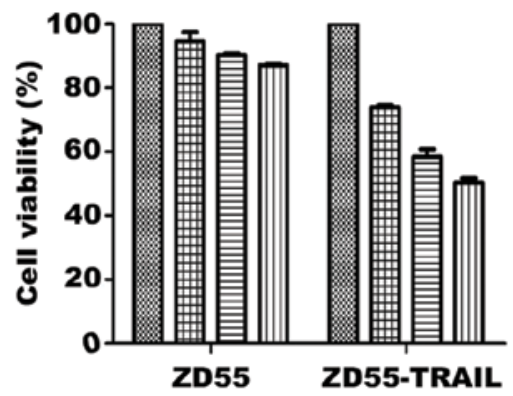

D

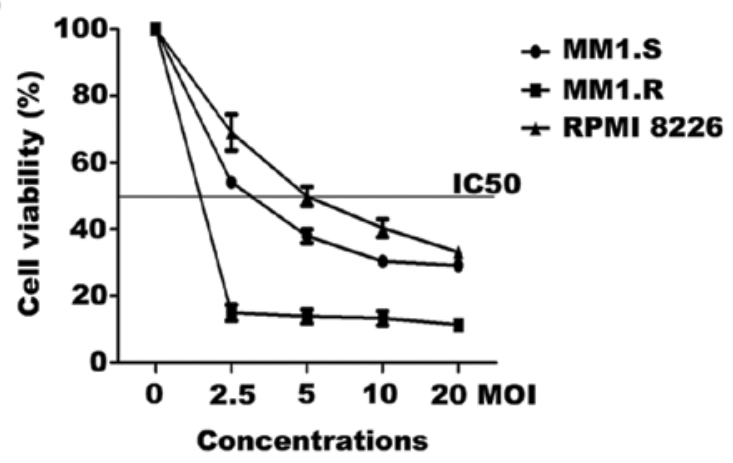

E
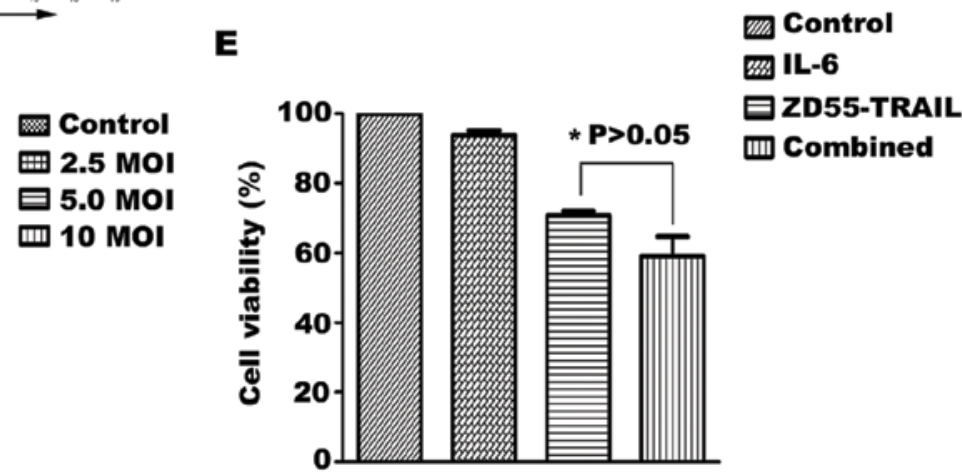

Figure 1. Infection and cytotoxicity of recombinant adenoviruses in MM cells. RPMI-8226 cells were treated with increasing concentrations of ZD55-EGFP (0-50 MOI) for 24 or $48 \mathrm{~h}$. (A) The cells were observed under a fluorescence microscope to detect GFP expression. (B) Treated cells were analyzed using a FACScan flow cytometer and CellQuest software. The percentage of cells with GFP (FL1-H, green fluorescence intensity) is shown. (C) RPMI-8226 cells were infected with ZD55 or ZD55-TRAIL at the indicated MOIs for $48 \mathrm{~h}$. Cell viability was evaluated by MTT assay. Bars represent means \pm SE, $\mathrm{n}=3$. (D) MM1.S, MM1.R and RPMI-8226 cells were treated with ZD55-TRAIL at the indicated doses for $48 \mathrm{~h}$. Viability was assessed by MTT assay. IC ${ }_{50}$ values for different MM cell lines are indicated. Points, mean of 3 separate experiments; bars, SE. (E) RPMI-8226 cells were cultured for 24 h with ZD55-TRAIL (8 MOI), IL-6 $(10 \mathrm{ng} / \mathrm{ml})$, or ZD55-TRAIL + IL-6 (Combined). Cell viability was measured by MTT assay. Data represent the means \pm SE of triplicate determinations. MM, multiple myeloma.

\section{Results}

ZD55 effectively infects MM cells and inhibits the growth of myeloma cell lines. To determine the infectivity of ZD55 in MM cells, RPMI-8226 cells were treated with the ZD55-EGFP vector at the indicated MOIs for $24 \mathrm{~h}$, and then GFP-positive cells were observed under a fluorescence microscopy. As shown in Fig. 1A, RPMI-8226 cells in culture were effectively infected with ZD55-EGFP in a dose-dependent manner. As determined by flow cytometric analysis, $52 \%$ GFP-positive cells were noted in the group treated with ZD55-EGFP at an MOI of 25 for $48 \mathrm{~h}$, while $62 \%$ GFP-positive cells were observed in the group treated with this virus at an MOI of 50 (Fig. 1B). To test whether ZD55-TRAIL has enhanced cytotoxicity, a cell viability assay was performed using RPMI-8226 cells infected with ZD55TRAIL or ZD55, respectively, at the indicated MOIs for $48 \mathrm{~h}$. The viability of RPMI-8226 cells was significantly reduced by ZD55-TRAIL in a dose-dependent manner when compared with the viability of the cells with no viral treatment, whereas ZD55 had a slight cytotoxic effect (Fig. 1C). Importantly, dosedependent growth inhibition was also observed in the MM1.S and the drug-resistant cell line MM1.R (Fig. 1D). Since IL-6 mediates growth and survival of MM cells, we next examined the effect of ZD55-TRAIL on RPMI-8226 cells in the presence of exogenous IL- 6 . We found that IL-6 $(10 \mathrm{ng} / \mathrm{ml}) \mathrm{did}$ not provide protection against ZD55-TRAIL-induced cell
A
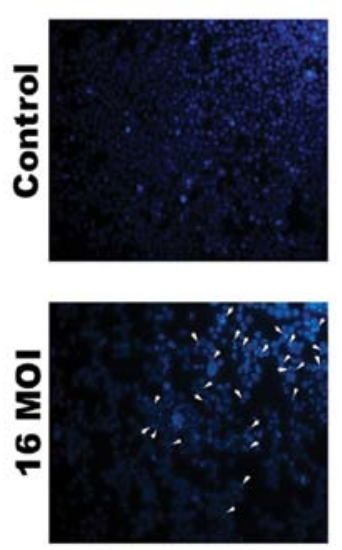

B

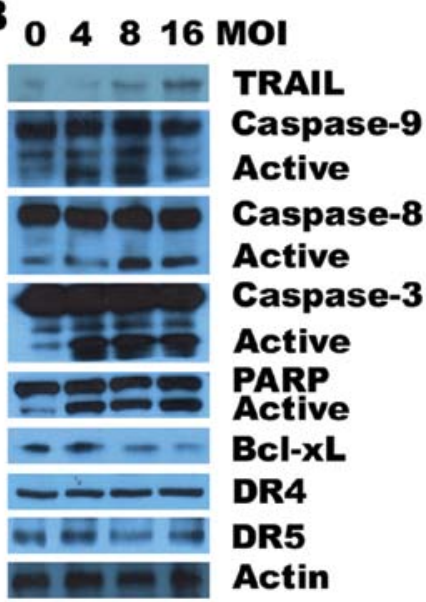

Figure 2. Treatment of ZD55-TRAIL results in the apoptosis of MM cells. (A) RPMI-8226 cells were infected with or without ZD55-TRAIL at an MOI of 16 for $24 \mathrm{~h}$ and were then stained with Hoechst 33258. Arrows indicate apoptotic nuclei. (B) After incubation of RPMI-8226 cells for $24 \mathrm{~h}$ with the indicated concentrations of ZD55-TRAIL, whole cell extracts were analyzed for activation of the caspase pathway, levels of DR4 and DR5, as well as TRAIL protein expression by western blot analysis. A representative of 3 separate experiments is shown. MM, multiple myeloma.

death (Fig. 1E), suggesting that ZD55-TRAIL, unlike Dex, is able to inhibit cytokine-induced MM cell growth. 
A

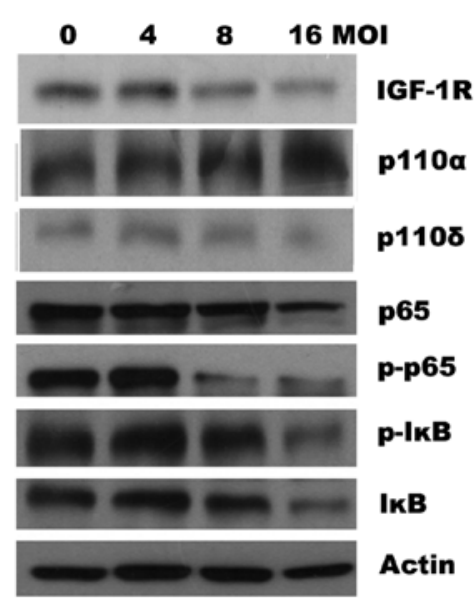

B

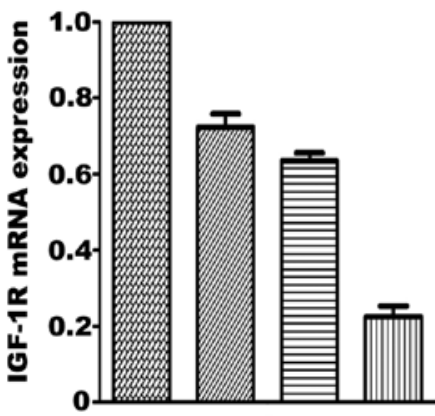

C

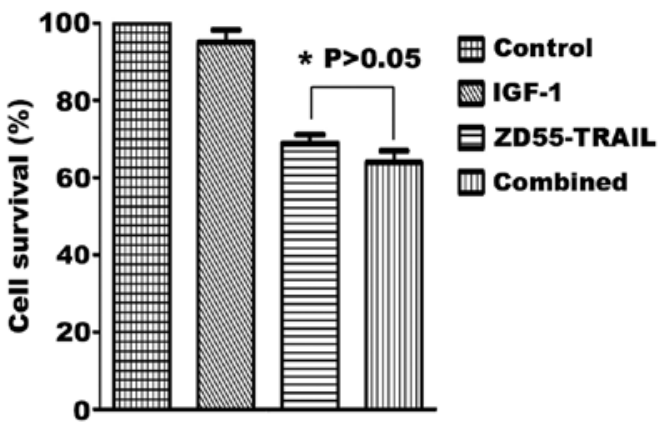

D

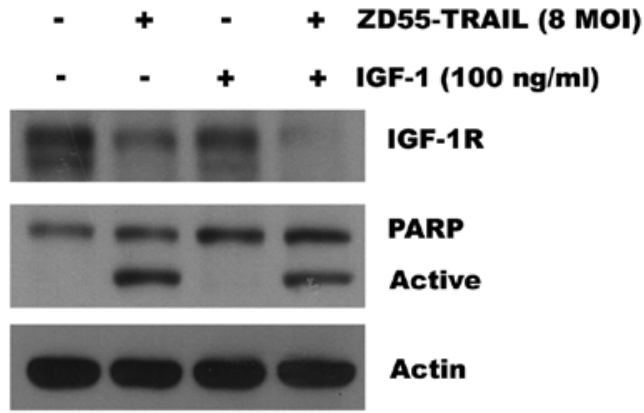

Figure 3. ZD55-TRAIL inhibits the expression of IGF1-R and NFkB pathway-related proteins in RPMI-8226 cells. (A) RPMI-8226 cells were treated with various concentrations of ZD55-TRAIL for $24 \mathrm{~h}$, and then whole cell extracts were analyzed for IGF1-R and expression of PI3K/NFKB pathway-related proteins by western blot analysis. The data are representative of 3 determinations with identical results. (B) After treatment of RPMI-8226 cells with ZD55-TRAIL (8 MOI) for the indicated times, total mRNA was prepared and analyzed for expression of IGF1-R by real-time PCR. Y axis indicates the relative expression of IGF1-R. Each point or bar represents the means \pm SE of 3 experiments. (C) The cells were incubated with ZD55-TRAIL (8 MOI), IGF-1 (100 ng/ml), or ZD55-TRAIL plus IGF-1 (combined) for $24 \mathrm{~h}$, and cell viability was examined by MTT colorimetric assay. *P>0.05 vs. ZD55-TRAIL (Student's t-test). (D). The treated cells were harvested after $24 \mathrm{~h}$. Whole cell lysates were subjected to western blotting for protein expression of IGF1-R and PARP. Anti- $\beta$-actin antibody was used as a control for protein loading. IGF1-R, insulin-like growth factor-1 receptor.

Inhibition of myeloma cell proliferation by ZD55-TRAIL is due to apoptosis induction. We next determined the effects of ZD55-TRAIL on induction of apoptosis by the quantification of apoptotic nuclei following Hoechst 33258 staining. As shown in Fig. 2A, ZD55-TRAIL significantly increased DNA fragmentation in RPMI-8226 cells, indicating that MM cells underwent apoptosis following ZD55-TRAIL infection. We further used immunoblotting to assess the expression of TRAIL and activation of caspases in the RPMI- 8226 cells treated with ZD55-TRAIL. ZD55-TRAIL at the tested concentration range (4-16 MOIs) effectively induced cleavage of caspase-8, -9 and -3 , followed by PARP cleavage, while the levels of TRAIL expression increased in a dose-dependent manner (Fig. 2B). However, during ZD55-TRAIL treatment, the expression levels of DR4 and DR5 were not affected. Previous studies suggest that $\mathrm{Bcl}-2$ or Bcl-xL confers resistance to chemotherapy in MM cells (18). We found that ZD55-TRAIL decreased the expression of $\mathrm{Bcl}-\mathrm{xL}$ in a dose-dependent fashion.

ZD55-TRAIL inhibits IGF-1R and may overcome drug resistance. It was recently reported that signaling through the IGF-1/IGF-1R axis contributes to acquired bortezomib resistance (19), and that inhibition of IGF-1R enhances cell death of MM cells induced by an IKK2 inhibitor (20). In the present study, we aimed to ascertain whether ZD55-TRAIL suppresses IGF-1R expression in RPMI-8226 cells. As shown in Fig. 3A, the virus decreased the level of IGF-1R as well as $\mathrm{NF \kappa B}$ pathway-related proteins, which included p-p65 and $\mathrm{p}-\mathrm{I} \kappa \mathrm{B}$, although the precise mechanism by which it does so is unknown. Moreover, quantitative PCR analysis showed that the mRNA expression of IGF-1R was also significantly inhibited by ZD55-TRAIL in a time-dependent manner (Fig. 3B). We next examined the effects of IGF-1 on ZD55-TRAIL-induced cell death. Exposure of MM cells to IGF-1 (100 ng/ml) did not reverse the cellular growth inhibition and apoptosis induced by ZD55-TRAIL (Fig. 3C and D) indicating that IGF-1R inhibition, by ZD55-TRAIL, may contribute to the enhanced cytotoxic action of the virus.

LY294002 has a synergistic effect with ZD55-TRAIL to inhibit cell proliferation in myeloma cells. Given the fact that the PI3K/AKT/mTOR pathway is constitutively activated in human MM cell lines and in primary plasmocytes from patients with MM, we next examined the effects of ZD55-TRAIL on several key signaling molecules in RPMI8226 cells. We found that ZD55-TRAIL activated AKT and mTOR as evidenced by the upregulation of phosphor-mTOR (Ser2448) and phosphorylated AKT at Ser473 (Fig. 4A), which may stimulate MM cell growth and promote cell survival and migration $(21,22)$. To improve the anti-myeloma efficacy of 
A

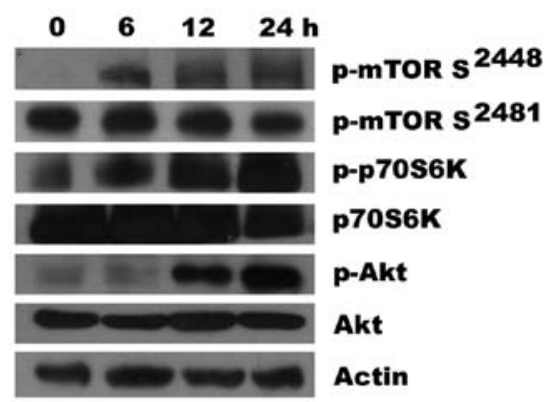

B
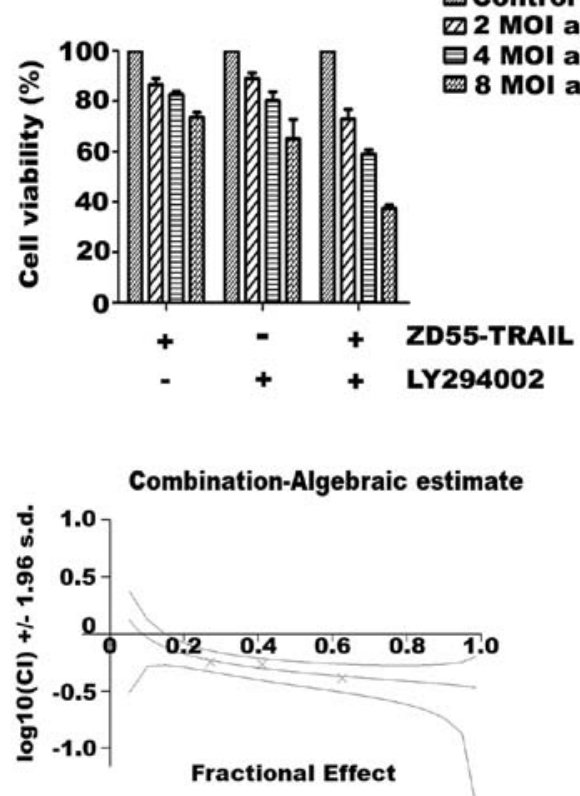

C

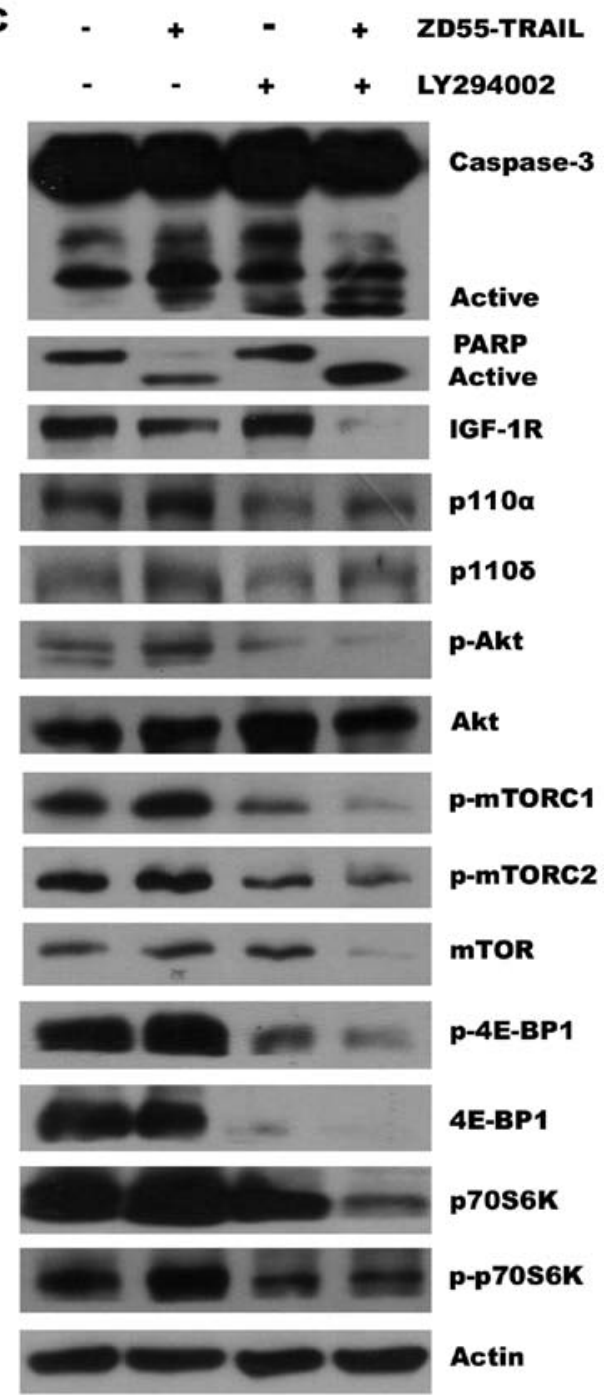

Figure 4. Combination of ZD55-TRAIL with LY294002 strongly inhibits the activation of the AKT/mTOR pathway and enhances cytotoxicity against MM cells. (A) RPMI-8226 cells were infected with ZD55-TRAIL (8 MOI) for the indicated times. Total cell lysates were analyzed by western blotting with antibodies against the phosphorylated form of mTOR on Ser2448 and Ser2481, phospho-AKT, AKT, phospho-p70S6K and p70S6K. Anti- $\beta$-actin was used as a loading control. (B) The cells were treated with increasing concentrations of ZD55-TRAIL and/or LY294002 for 24 h, and cell viability was determined by an MTT assay. Data are presented as the means \pm SE of 3 independent experiments. The combination index (CI) values calculated by CalcuSyn software are shown (lower panel). (C) RPMI-8226 cells were infected for $24 \mathrm{~h}$ with or without ZD55-TRAIL ( 8 MOI), in the presence or absence of LY294002 (20 $\mu$ M) and then total cell lysates were prepared for the detection of the activation of caspase-3 and cleavage of PARP by western blot analysis. The expression and phosphorylation of PI3K/AKT/mTOR proteins, and its downstream targets were also examined. MM, multiple myeloma.

ZD55-TRAIL, RPMI-8226 cells were treated with a series of doses of ZD55-TRAIL or/and LY294002, a PI3K inhibitor. As revealed in Fig. 4B, treatment of MM cells with ZD55-TRAIL plus LY294002 resulted in significantly lower cell viability than that following either ZD55-TRAIL or LY294002 treatment. By combination index analysis, we observed synergistic cytotoxic effects when the two agents were combined, as evidenced by a CI $<0$ (Fig. 4B). Furthermore, western blot analysis showed that addition of the PI3K inhibitor LY294002 led to increased levels of cleaved forms of caspase- 3 and PARP (Fig. 4C), suggesting enhanced apoptosis upon co-treatment. To understand the mechanisms involved in the apoptotic synergy between ZD55-TRAIL and LY294002, we examined the effects of the combination therapy on mTOR, a member of the PI3K-related kinase protein family. Western blot analysis indicated that LY294002 suppressed mTOR expression and phosphorylation of mTOR protein on Ser2448, a marker for the presence of mammalian target of rapamycin complex 1 (mTORC1) (23), as well as phosphorylation of mTOR on Ser2481, a marker for mTORC2 activity $(23,24)$. As a result, phosphorylation of 4E-BP1 and p70S6K, downstream targets of mTORC1, as well as phospho-AKT (Ser473), a downstream target of mTORC2 were also inhibited by LY294002. Notably, enhanced inhibition of key components of the mTOR pathway was observed in the cells treated with ZD55-TRAIL combined with LY294002 (Fig. 4C).

MG132 upregulates DR5 and potentiates ZD55-TRAILmediated cell death in RPMI-8226 cells. Since proteasome inhibitor MG132 is shown to sensitize tumor cells to TRAIL via upregulation of DR4 and DR5 $(25,26)$, we therefore examined the synergistic effects of ZD55-TRAIL in combination with MG132. For this purpose, we treated RPMI-8226 cells with different concentrations of MG132 
A

O $160320480 \mathrm{nM}$

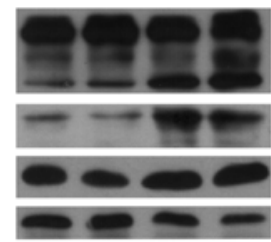

PARP

Active

DR5

DR4

Actin

$\mathbf{B}$
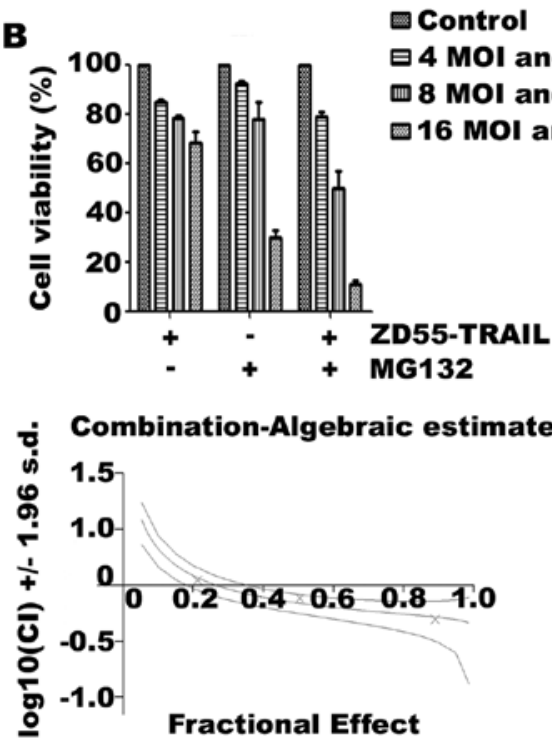

C

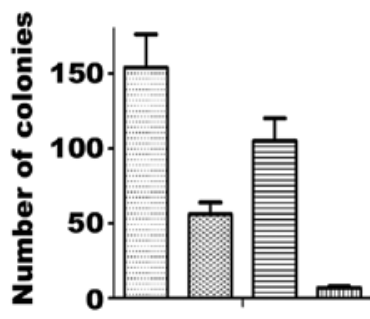

$\square$ Control
D

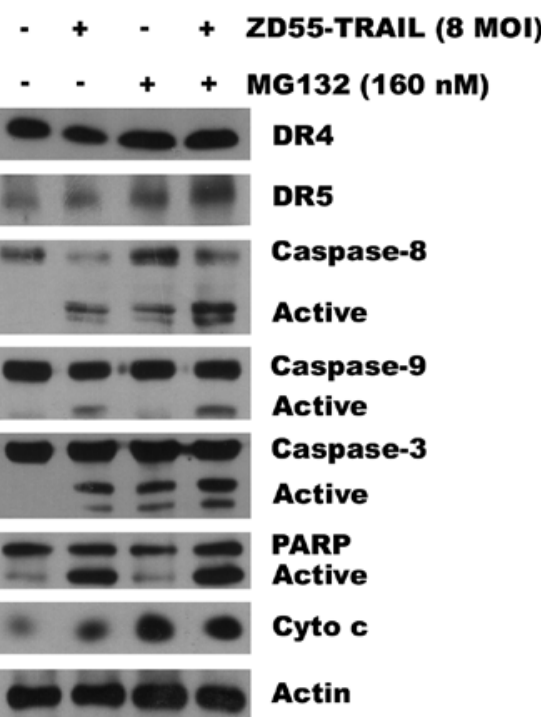

E

O ZD55-TRAIL

घ MG-132

(I) Combined

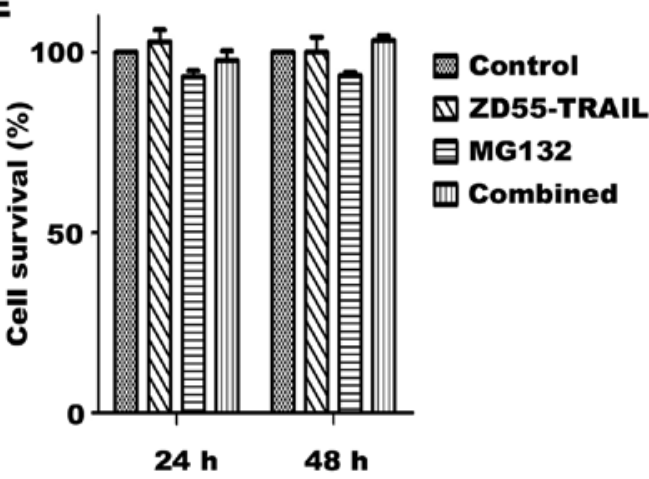

Figure 5. Mechanism of the sensitization to ZD55-TRAIL-induced apoptosis by MG132 in RPMI-8226 cells. (A) Cleavage of PARP and protein expression of DR4, DR5 and $\beta$-actin were assessed in RPMI-8226 cells treated with MG132 at the indicated doses by immunoblotting. (B) RPMI-8226 cells were plated in triplicate and concurrently treated with ZD55-TRAIL (up to $16 \mathrm{MOI}$ ) and MG132 (up to $320 \mathrm{nM}$ ) for $24 \mathrm{~h}$. MTT assays were performed to assess growth inhibition. Combination index values for each fraction affected are shown (lower panel). (C) RPMI-8226 cells were treated with a low-dose (8 MOI) of ZD55-TRAIL and/or MG132 $(160 \mathrm{nM})$. The inhibitory effect of colony formation of myeloma cells was examined by colony forming cell assay. Columns, mean of 3 separate experiments; bars, SE. (D) Cells were incubated for $24 \mathrm{~h}$ with or without ZD55-TRAIL and MG132 as indicated. Cell lysates were fractionated on 12\% SDSpolyacrylamide gels and analyzed by western blotting with antibodies against DR4, DR5, cyto $c$ and caspase pathway-related proteins. (E) L02 cells were treated with ZD55-TRAIL (8 MOI) or MG132 $(160 \mathrm{nM})$, or a combination of the two agents for $24 \mathrm{~h}$ and then analyzed by MTT assay for growth inhibition. The results are representative of 3 independent experiments. Bars represent means \pm SE. DR4, death receptor 4; DR5, death receptor 5; cyto $c$; cytochrome $c$.

and ZD55-TRAIL either alone or in combination for $24 \mathrm{~h}$ and assessed cell viability using an MTT assay. We found that a low-dose of MG132 (160-320 nM) markedly enhanced the cell death of low-dose ZD55-TRAIL (8-16 MOIs)-infected RPMI-8226 cells (Fig. 5A). The combination index values indicated a strong synergy between MG132 and ZD55TRAIL (Fig. 5B). Furthermore, ZD55-TRAIL and MG132 reduced the colony formation ability of MM cells in vitro, which was significantly increased when the two treatments were combined (Fig. 5C). Analysis of RPMI-8226 nuclei after Hoechst staining revealed that the synergistic cytotoxic effect of the combination therapy was due to enhanced apoptosis (data not shown), which was confirmed by western blotting that showed an enhanced caspase cascade activation in the cells treated with the two agents as compared with the ZD55-TRAIL- or MG132-treated cells (Fig. 5D). To assess the effects of ZD55-TRAIL combined with MG132 on normal cells, we treated L02, a normal human liver cell line, with ZD55-TRAIL, MG132, or ZD55-TRAIL plus MG132 and then determined the cell viability using an MTT assay. As shown in Fig. 5E, ZD55-TRAIL, MG132, or the combined treatment at the indicated dosage did not induce cytotoxicity against normal cells.

\section{Discussion}

The oncolytic adenovirus is a potent member of a potentially new class of antitumor agents that has been previously demonstrated to have activity in MM (27-29). Among the viruses used for virotherapy of MM, different serotypes of adenovi- 
ruses have been studied, with the goal of finding a translational application for the treatment of myeloma progressing on prior therapy $(30,31)$. In this regard, Senac et al showed that adenovirus serotype 5 (Ad5) infected most MM cell lines and primary myeloma cells from patients and that oncolytic Ad5 appeared to infect $\mathrm{CD} 138^{+} \mathrm{MM}$ cells 10 - to 70 -fold more efficiently than normal marrow cells (30), suggesting the possibility of translating adenovirus-based virotherapies to MM patients. In the present study, we show that ZD55, an Ad5 oncolytic virus, efficiently infected the MM cell line RPMI-8226, as demonstrated by fluorescence microscopy and flow cytometric analysis. Using ZD55-TRAIL as a gene virotherapy, we found that the virus was able to effectively kill a panel of myeloma cell lines, which included a drug-resistant cell line MM1.R. Consistent with previous reports on hepatocellular carcinoma cell lines $(15,16)$, treatment of RPMI-8226 cells with ZD55TRAIL resulted in induction of apoptosis. This effect was also confirmed by our observed cleavage of caspase-3 and PARP. In addition, we found that IL-6 failed to protect against ZD55TRAIL-induced growth inhibition. Therefore, ZD55-TRAIL, which kills MM cells via both oncolysis and apoptosis, may be particularly useful to overcome drug resistance.

The proliferation and survival of MM cells have been shown to be related to the activation of several signaling pathways such as PI3K/AKT, JAK/STAT3, MAPK/ERK and NFKB (32-34), which can be stimulated by several cytokines including IL-6 and IGF-1 (35). In MM, IGFs and IGF-1R signaling was correlated with stimulation of proliferation, survival and drug-resistance $(36,37)$. Recent studies suggest that the inhibition of IGF-1R decreases cell proliferation. Furthermore, combinations of multiple drugs such as the IKK2 inhibitor AS602868 and bortezomib with agents intervening in the IGF-1R pathway were found to result in enhanced apoptosis and reversal of drug-resistance $(19,20,38)$, suggesting that targeting the IGF-1/IGF-1R axis is a promising approach for the treatment of MM. Our results confirm the inhibitory effect of ZD55-TRAIL on the expression of IGF-1R in RPMI8226 cells. Moreover, we found that the virus significantly inhibited the NFKB pathway in MM cells, as assessed by ZD55-TRAIL-induced downregulation of phosphorylation and expression of the p65 subunit of $\mathrm{NF} \kappa \mathrm{B}$ and inhibitory $\kappa \mathrm{B}(\mathrm{I} \kappa \mathrm{B})$. It is noteworthy that RPMI-8226 cells were more sensitive to ZD55-TRAIL-induced cell death than MM1.S and MM1.R cells. This result is consistent with a previous finding that RPMI-8226 cells express more IGF-1R than MM.1S and $\mathrm{U} 266$ cells, and that the cytotoxic effect of anti-IGF-1R is more effective on MM cells with a high level of IGF-1R (20). Our data also showed that ZD55-TRAIL-mediated apoptosis and cell growth inhibition were not reversed by IGF. Thus, ZD55-TRAIL can be considered as an efficient anticancer agent acting through diverse mechanisms of action in the killing of MM cells by downregulation of IGF-1R.

Prior studies have shown that adenoviral infection triggers the activation of PI3K and its downstream molecules such as AKT, ERK1/2, JNK and MAPK by viral fiber binding with the Coxsackie-adenovirus receptor (39-41), which may benefit tumor cell survival. In the present study, mechanistic analyses in RPMI-8226 cells showed a high level of phosphorylation of mTOR on Ser2448, which represents the activity of mTORC1, and downstream activation of AKT and p70S6K in
ZD55-TRAIL-treated cells, which increased in a dose-dependent manner. These data suggest that the virus elicited the activation of the AKT/mTOR pathway. Based on these results, we investigated the effect of ZD55-TRAIL combined with the AKT inhibitor LY294002 on MM cells. Our results revealed that the combination of LY294002 together with ZD55TRAIL synergistically inhibited cell proliferation and induced apoptosis via the inhibition of the AKT/mTOR pathway. To our knowledge, this is the first report demonstrating that an oncolytic adenovirus synergizes with an AKT inhibitor to induce death of MM cells.

TRAIL, which induces apoptosis via interactions with its death receptors DR4 and DR5, represents a promising candidate agent for the treatment of MM $(11,42,43)$. Recent studies suggest that there is a correlation between TRAIL sensitivity and death receptor expression in cancer cells (44), thus the upregulation of death receptor expression renders MM cells more sensitive to the action of TRAIL. In the present study, we showed that increased expression of DR5, but not DR4, induced by MG132 was associated with ZD55-TRAIL sensitivity in RPMI-8226 cells. This is consistent with a previous report showing that overexpression of DR5 in TRAIL-resistant cancer cells restored TRAIL sensitivity (45). In addition, we observed that MG132 and ZD55-TRAIL combination therapy significantly inhibited the clonogenic survival of MM cells, which indicates the importance of the combination therapy in the elimination of cancer progenitor cells.

In summary, our results showed that ZD55-TRAIL induced significant cytotoxicity in drug-sensitive and drug-resistant myeloma cell lines in vitro. In addition to an oncolytic effect, ZD55-TRAIL induced apoptosis through activation of the caspase pathway. Furthermore, we showed for the first time that ZD55-TRAIL markedly inhibited the expression of IGF-1R and downstream NFKB. The combination of ZD55-TRAIL and LY294002 in RPMI-8226 cells overcame the activation of mTOR and AKT induced by the virus and consequently promoted cell death. Moreover, increased expression of DR5 enhanced the apoptotic response to the combined treatment of MG132 and ZD55-TRAIL. These findings provide the rationale for further pre-clinical evaluation of TRAIL-based adenoviral therapy in combination with a PI3K inhibitor or a proteosome inhibitor for MM.

\section{Acknowledgements}

The present study was supported by the National Natural Science Foundation of China grants (nos. 81370646, 81370645 and 81302036), the Doctoral Fund of the Ministry of Education of China (no. 20120101110010), the Funds of the Science Technology Department of Zhejiang Province (no. 2012C13021-2), the Fund of the Health Bureau of Zhejiang Province (no. 2010SSA006), the Natural Science Foundation of Zhejiang Province (no. Y2090455), and the Foundation of the Zhejiang Municipal Commission of Population and Family Planning (no. 2009-74).

\section{References}

1. Kyle RA: Multiple myeloma: an odyssey of discovery. Br J Haematol 111: 1035-1044, 2000. 
2. Brenner H, Gondos A and Pulte D: Expected long-term survival of patients diagnosed with multiple myeloma in 2006-2010. Haematologica 94: 270-275, 2009.

3. Barlogie B, Attal M, Crowley J, et al: Long-term follow-up of autotransplantation trials for multiple myeloma: update of protocols conducted by the Intergroupe Francophone du Myelome, Southwest Oncology Group, and University of Arkansas for Medical Sciences. J Clin Oncol 28: 1209-1214, 2010.

4. Hideshima T and Anderson KC: Molecular mechanisms of novel therapeutic approaches for multiple myeloma. Nat Rev Cancer 2: 927-937, 2002

5. Hellwig CT and Rehm M: TRAIL signaling and synergy mechanisms used in TRAIL-based combination therapies. Mol Cancer Ther 11: 3-13, 2012.

6. Herbst RS, Eckhardt SG, Kurzrock R, et al: Phase I dose-escalation study of recombinant human Apo2L/TRAIL, a dual proapoptotic receptor agonist, in patients with advanced cancer. J Clin Oncol 28: 2839-2846, 2010.

7. Gajate $\mathrm{C}$ and Mollinedo F: Edelfosine and perifosine induce selective apoptosis in multiple myeloma by recruitment of death receptors and downstream signaling molecules into lipid rafts. Blood 109: 711-719, 2007.

8. Mitsiades CS, Treon SP, Mitsiades N, et al: TRAIL/Apo2L ligand selectively induces apoptosis and overcomes drug resistance in multiple myeloma: therapeutic applications. Blood 98: 795-804, 2001.

9. Gazitt Y: TRAIL is a potent inducer of apoptosis in myeloma cells derived from multiple myeloma patients and is not cytotoxic to hematopoietic stem cells. Leukemia 13: 1817-1824, 1999.

10. Jazirehi AR, Ng CP, Gan XH, Schiller G and Bonavida B Adriamycin sensitizes the adriamycin-resistant 8226/Dox40 human multiple myeloma cells to Apo2L/tumor necrosis factorrelated apoptosis-inducing ligand-mediated (TRAIL) apoptosis. Clin Cancer Res 7: 3874-3883, 2001

11. Vitovski S, Chantry AD, Lawson MA and Croucher PI: Targeting tumour-initiating cells with TRAIL based combination therapy ensures complete and lasting eradication of multiple myeloma tumours in vivo. PLoS One 7: e35830, 2012.

12. Hermiston TW and Kuhn I: Armed therapeutic viruses: strategies and challenges to arming oncolytic viruses with therapeutic genes. Cancer Gene Ther 9: 1022-1035, 2002.

13. Zhang ZL, Zou WG, Luo CX, et al: An armed oncolytic adenovirus system, ZD55-gene, demonstrating potent antitumoral efficacy. Cell Res 13: 481-489, 2003.

14. Dong F, Wang L, Davis JJ, et al: Eliminating established tumor in $\mathrm{nu} / \mathrm{nu}$ nude mice by a tumor necrosis factor- $\alpha$-related apoptosisinducing ligand-armed oncolytic adenovirus. Clin Cancer Res 12: 5224-5230, 2006

15. Pei Z, Chu L, Zou W, et al: An oncolytic adenoviral vector of Smac increases antitumor activity of TRAIL against HCC in human cells and in mice. Hepatology 39: 1371-1381, 2004.

16. Wang SB, Tan Y, Lei W, et al: Complete eradication of xenograft hepatoma by oncolytic adenovirus ZD55 harboring TRAILIETD-Smac gene with broad antitumor effect. Hum Gene Ther 23: 992-1002, 2012

17. Meng H, Jin Y, Liu H, You L, Yang C, Yang X and Qian W: SNS-032 inhibits mTORC1/mTORC2 activity in acute myeloid leukemia cells and has synergistic activity with perifosine against Akt. J Hematol Oncol 6: 18, 2013.

18. Chauhan D, Velankar M, Brahmandam M, et al: A novel Bcl-2/Bcl-X $/$ / Bcl-w inhibitor ABT-737 as therapy in multiple myeloma. Oncogene 26: 2374-2380, 2007.

19. Kuhn DJ, Berkova Z, Jones RJ, et al: Targeting the insulin-like growth factor-1 receptor to overcome bortezomib resistance in preclinical models of multiple myeloma. Blood 120: 3260-3270, 2012.

20. Tagoug I, Sauty De Chalon A and Dumontet C: Inhibition of IGF-1 signalling enhances the apoptotic effect of AS602868, an IKK2 inhibitor, in multiple myeloma cell lines. PLoS One 6: e22641, 2011.

21. Ikeda H, Hideshima T, Fulciniti M, et al: PI3K/p1108 is a novel therapeutic target in multiple myeloma. Blood 116: 1460-1468, 2010.

22. Maiso P, Liu Y, Morgan B, et al: Defining the role of TORC1/2 in multiple myeloma. Blood 118: 6860-6870, 2011.

23. Park S, Chapuis N, Tamburini J, et al: Role of the PI3K/AKT and mTOR signaling pathways in acute myeloid leukemia. Haematologica 95: 819-828, 2010.
24. Oh WJ and Jacinto E: mTOR complex 2 signaling and functions. Cell Cycle 10: 2305-2316, 2011.

25. Hougardy BM, Maduro JH, van der Zee AG, de Groot DJ, van den Heuvel FA, de Vries EG and de Jong S: Proteasome inhibitor MG132 sensitizes HPV-positive human cervical cancer cells to rhTRAIL-induced apoptosis. Int J Cancer 118: 1892-1900, 2006

26. Seol DW: p53-Independent up-regulation of a TRAIL receptor DR5 by proteasome inhibitors: a mechanism for proteasome inhibitor-enhanced TRAIL-induced apoptosis. Biochem Biophys Res Commun 416: 222-225, 2011.

27. Bais S, Bartee E, Rahman MM, McFadden G and Cogle CR Oncolytic virotherapy for hematological malignancies. Adv Virol 2012: 186512,2012.

28. Raus S, Coin S and Monsurrò V: Adenovirus as a new agent for multiple myeloma therapies: opportunities and restrictions. Korean J Hematol 46: 229-238, 2011.

29. Naik S, Nace R, Federspiel MJ, Barber GN, Peng KW and Russell SJ: Curative one-shot systemic virotherapy in murine myeloma. Leukemia 26: 1870-1878, 2012.

30. Senac JS, Doronin K, Russell SJ, Jelinek DF, Greipp PR and Barry MA: Infection and killing of multiple myeloma by adenoviruses. Hum Gene Ther 21: 179-190, 2010.

31. Chen CY, Senac JS, Weaver EA, et al: Species D adenoviruses as oncolytics against B-cell cancers. Clin Cancer Res 17: 6712-6722, 2011.

32. Catlett-Falcone R, Landowski TH, Oshiro MM, et al: Constitutive activation of Stat 3 signaling confers resistance to apoptosis in human U266 myeloma cells. Immunity 10: 105-115, 1999.

33. Tu Y, Gardner A and Lichtenstein A: The phosphatidylinositol 3-kinase/AKT kinase pathway in multiple myeloma plasma cells: roles in cytokine-dependent survival and proliferative responses. Cancer Res 60: 6763-6770, 2000.

34. Hsu J, Shi Y, Krajewski S, et al: The AKT kinase is activated in multiple myeloma tumor cells. Blood 98: 2853-2855, 2001.

35. Bruno B, Giaccone L, Rotta M, Anderson K and Boccadoro M Novel targeted drugs for the treatment of multiple myeloma: from bench to bedside. Leukemia 19: 1729-1738, 2005.

36. Sprynski AC, Hose D, Caillot L, et al: The role of IGF-1 as a major growth factor for myeloma cell lines and the prognostic relevance of the expression of its receptor. Blood 113: 4614-4626, 2009.

37. Huo J, Xu S, Lin B, Chng WJ and Lam KP: Fas apoptosis inhibitory molecule is upregulated by IGF-1 signaling and modulates Akt activation and IRF4 expression in multiple myeloma. Leukemia 27: 1165-1171, 2013.

38. Strömberg T, Ekman S, Girnita L, et al: IGF-1 receptor tyrosine kinase inhibition by the cyclolignan PPP induces $G_{2} / M$-phase accumulation and apoptosis in multiple myeloma cells. Blood 107: 669-678, 2006.

39. Thaci B, Ulasov IV, Wainwright DA and Lesniak MS: The challenge for gene therapy: innate immune response to adenoviruses. Oncotarget 2: 113-121, 2011.

40. Tamanini A, Nicolis E, Bonizzato A, Bezzerri V, Melotti P, Assael BM and Cabrini G: Interaction of adenovirus type 5 fiber with the coxsackievirus and adenovirus receptor activates inflammatory response in human respiratory cells. J Virol 80: 11241-11254, 2006.

41. Verdino P, Witherden DA, Havran WL and Wilson IA: The molecular interaction of CAR and JAML recruits the central cell signal transducer PI3K. Science 329: 1210-1214, 2010.

42. David E, Sinha R, Chen J, Sun SY, Kaufman JL and Lonial S: Perifosine synergistically enhances TRAIL-induced myeloma cell apoptosis via up-regulation of death receptors. Clin Cancer Res 14: 5090-5098, 2008

43. Surget $\mathrm{S}$, Chiron D, Gomez-Bougie P, et al: Cell death via DR5, but not DR4, is regulated by p53 in myeloma cells. Cancer Res 72: 4562-4573, 2012.

44. Voortman J, Resende TP, Abou El Hassan MA, Giaccone G and Kruyt FA: TRAIL therapy in non-small cell lung cancer cells: sensitization to death receptor-mediated apoptosis by proteasome inhibitor bortezomib. Mol Cancer Ther 6: 2103-2112, 2007.

45. Mitsiades N, Poulaki V, Mitsiades C and Tsokos M: Ewing's sarcoma family tumors are sensitive to tumor necrosis factorrelated apoptosis-inducing ligand and express death receptor 4 and death receptor 5. Cancer Res 61: 2704-2712, 2001. 